\title{
ANGLE-RESOLVED FLUORESCENCE DEPOLARIZATION OF MACROSCOPICALLY ORDERED BILAYERS OF UNSATURATED LIPIDS
}

\author{
MARTIN J.M. VAN DE VEN and YEHUDI K. LEVINE * \\ Biophysics Department, Physics Laboratory, University of Utrecht, Princetonplein 5, 3584 CC Utrecht (The Netherlands)
}

(Received April 19th, 1984)

Key words: Lipid bilayer; Unsaturated lipid; Order parameter; Fluorescence depolarization; Diphenylhexatriene

The orientational order and dynamics of diphenylhexatriene molecules incorporated in planar multibilayers of unsaturated lipids were studied by angle-resolved fluorescence depolarization at room temperature. Bilayer systems of the phosphatidylcholines soybean PC, dioleoyl PC and egg PC as well as the galactolipid digalactosyl diacylglycerol (DGDG) were used in the experiments. The depolarization ratios of diphenylhexatriene in these systems exhibited a weak angular dependence, in marked contrast to those observed from diphenylhexatriene in multibilayers of saturated phosphatidylcholines (Vos, M.H., Kooyman, R.P.H. and Levine, Y.K. (1983) Biochem. Biophys. Res. Commun. 116, 462-468). The results indicate that the diphenylhexatriene molecules possess a low degree of orientational order. However, the dynamics of the reorientational motions are inconsistent with the assumption that the diphenylhexatriene molecules possess effective cylindrical symmetry about their long axes. The fluorescence depolarization experiment is sensitive to the rotational motions about the long axes as the absorption and emission moments of diphenylhexatriene molecules are not mutually parallel. It is suggested that diphenylhexatriene molecules in bilayers of unsaturated lipids undergo slower reorientational motions around their long molecular axes than in bilayers of saturated phosphatidylcholines. A further conclusion of this study is that the 'wobbling-in-cone' model provides a poor description of the behaviour of diphenylhexatriene molecules in lipid bilayers.

\section{Introduction}

In recent years there has been a growing interest in the application of fluorescence depolarization experiments to studies of molecular order and dynamics in biological membranes and lipid bilayers. In these experiments fluorescent reporter molecules such as 1,6-diphenyl-1,3,5-hexatriene (DPH) are incorporated into the membranes and their fluorescence depolarization studied. The orientational distribution of the molecules in the membranes is commonly described in terms of the order parameters $\left\langle P_{2}\right\rangle=\frac{1}{2}\left\langle 3 \cos ^{2} \beta-1\right\rangle$ and

\footnotetext{
* To whom correspondence should be addressed.
}

$\left\langle P_{4}\right\rangle=\frac{1}{8}\left\langle 35 \cos ^{4} \beta-30 \cos ^{2} \beta+3\right\rangle$, where the angled brackets denote an ensemble average and $P_{L}$ is the Legendre polynomial of order $L$. The dynamics of the probe molecules is characterized in terms of correlation times whose physical significance depends on the particular model of motion used in the analysis.

In two recent papers from our laboratory [1-3] we described a new type of steady-state fluorescence depolarization experiment suitable for studies of macroscopically ordered lipid bilayer systems. In this experiment the fluorescence depolarization ratios are determined as a function of two independent angles: (1) the angle of incidence of light measured relative to the normal to the lamellar surface and (2) the angle between the directions 
of incidence and observation.

We have shown both theoretically [1] and experimentally $[2,3]$ that this type of angle-resolved fluorescence depolarization experiment (AFD) yields both the order parameters $\left\langle P_{2}\right\rangle$ and $\left\langle P_{4}\right\rangle$ as well as information about molecular dynamics and the mutual orientation of the absorption and emission moments for molecules with effective cylindrical symmetry such as DPH. This method can thus be considered to be an alternative to time-resolved experiments on lipid vesicles [17].

AFD experiments on bilayers of saturated phosphatidylcholines, with or without cholesterol, have demonstrated the validity of this approach $[2,3]$. It was shown that a knowledge of both $\left\langle P_{2}\right\rangle$ and $\left\langle P_{4}\right\rangle$ is often necessary in order to describe adequately the ordering and preferential orientation of the DPH molecules in the membrane systems. An important finding was that the absorption and emission moments of DPH are not mutually parallel as is commonly assumed. Rather, the angle between the moments depends on the bilayer system in which the DPH molecules are embedded. The rotational correlation times of DPH in these bilayers were determined on the basis of the strong collision model [1,5-7].

Here we report an extension of these studies to bilayers of the unsaturated lipids egg phosphatidylcholine, soybean phosphatidylcholine, dioleoylphosphatidylcholine and digalactosyl diacylglycerol containing DPH molecules. It is shown that in these systems the angle dependence of the polarization ratios is significantly different from that observed in bilayers of saturated lipids and reported in Refs. 2 and 3. An analysis of the data reveals that the introduction of unsaturation into the lipid chains has a strong influence on the reorientational dynamics of the DPH molecules, but causes only qualitative changes in their static orientational order as reflected in the order parameters $\left\langle P_{2}\right\rangle$ and $\left\langle P_{4}\right\rangle$.

Firstly, we give a short recapitulation of the theory and pay particular attention to three models for the reorientational motion (strong collision, rotational diffusion and 'wobbling-in-cone') which are used in the interpretation of the experimental data. Secondly, experimental AFD results are presented and discussed in the light of our earlier results. Analysis of the data indicates that the 'wobbling-in-cone' model, in contrast to the strong collision and rotational diffusion models, yields a poor representation of the reorientational dynamics of DPH molecules in lipid bilayers. Finally, it is argued that DPH molecules are not particularly suitable as probes for monitoring order and dynamics in unsaturated lipid bilayers.

\section{Theory}

Consider a macroscopically ordered membrane system containing fluorescent 'reporter' molecules and subjected to continuous illumination with a well-defined wavelength and polarization direction. The geometrical arrangement of the experiment is shown in Fig. 1. We define an axis system $(X Y Z)$ such that $X$ and $Y$ are in the membrane plane and $Z$ is parallel to the optical axis (director)

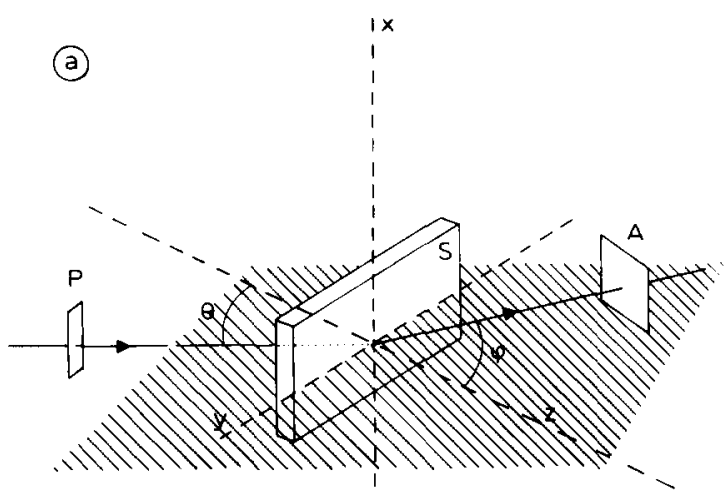

(b)

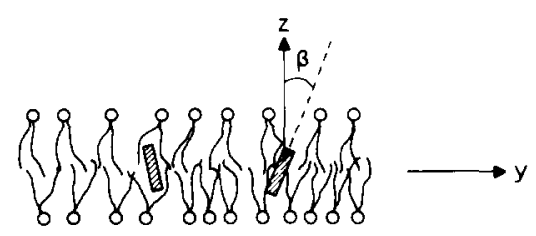

Fig. 1. (a) Experimental AFD geometry for a lamellar bilayer sample $S$ lying in the $X Y$ plane. $\theta$ and $\phi$ are the angles in air between the macroscopic director $Z$ and, respectively, the direction of excitation and observation of the fluorescence. The states of polarization, either parallel or perpendicular to the $Y Z$ plane are determined by the polarizer $P$ and the analyzer $A$. The angles $\theta$ and $\phi$ (taken to be positive, as drawn in the figure) change sign on rotation of either $P$ or $A$ through the $Z$-axis. (b) A cylindrically symmetric probe molecule, embedded in one of the approx. 1000 stacked bilayers in sample $S$, has an instantaneous angle $\beta$ relative to the director $Z$. 
of the membrane system. The angle $\theta$ corresponds to the direction of the incident (exciting) beam relative to the director of the membrane system. The angle $\phi$ is defined as the angle under which the light emission is observed. The principal direction of the polarizer $P$ can be set either perpendicular or parallel to the $Z Y$ plane.

The same holds for the emitted light where the analyzer $\mathrm{A}$ is placed in the path of the outgoing beam. Thus, four combinations of polarized fluorescence intensities $I_{\text {if }}$ can be measured: $I_{\mathrm{o}}, I_{\mathrm{oc}}$, $I_{\mathrm{eo}}$ and $I_{\mathrm{ee}}$ where the suffices o and e denote light polarized perpendicular to the $Z$-axis ('ordinary' beam) and light polarized in the $Z Y$-plane ("extraordinary' beam), respectively. In order to eliminate the dependence of $I_{\text {if }}$ on unknown quantities such as the incident light intensity, illuminated volume and absorption coefficients, only the depolarization ratios $R_{\mathrm{o}}=I_{\mathrm{oe}} / I_{\mathrm{oo}}$ and $R_{\mathrm{e}}=I_{\mathrm{eo}} / I_{\mathrm{ee}}$ are determined experimentally. It has been previously shown [1] that these polarization ratios are given by the relations:

$$
\begin{aligned}
R_{\mathrm{o}}= & R_{\mathrm{o}}(\phi)=\left[1-R_{1}+\left(R_{1}+R_{2}\right) \sin ^{2} \phi\right] /\left(1+R_{1}\right) \\
R_{\mathrm{e}}= & R_{\mathrm{e}}(\theta, \phi)=\left[1-R_{1}+\left(R_{3}+R_{1}\right) \sin ^{2} \theta\right] \\
& \times\left[1+R_{1}+\left(R_{2}-R_{1}\right) \sin ^{2} \phi+\left(R_{3}-R_{1}\right) \sin ^{2} \theta\right. \\
& \left.+R_{5} \sin ^{2} \theta \sin ^{2} \phi+R_{4} \sin 2 \theta \cdot \sin 2 \phi\right]^{-1}
\end{aligned}
$$

The only assumption underlying the derivation of Eqn. 1 is that the probe molecules are macroscopically distributed uniaxially about the normal to the surface of the sample. It can be shown further that Eqns. 1a and $1 \mathrm{~b}$ are obtained even if several independent probe populations are present, provided only that the total macroscopic distribution is uniaxially symmetric.

The parameters $R_{1}, \ldots, R_{5}$ contain all the information about the orientational order and dynamics of the fluorescent 'reporter' molecules in the membrane system. These parameters can only be interpreted on the basis of a model for the reorientational motion of the molecules. In addition, the molecular geometry must be known. In the analysis we shall assume that even if several independent probe molecule populations are present, they can all be described by the same dynamic behaviour.
The DPH molecules used in this study will be considered to possess an effective cylindrical symmetry. Furthermore, the absorption transition moment, $\mu$, will be taken to be parallel to the molecular symmetry axis. The emission transition moment, $\nu$, will, however, be allowed to make an angle $\beta_{v}$, relative to that axis. The angle $\beta_{v}$, will be considered further to be independent of the orientational and dynamic behaviour of the molecules.

With these conditions Eqns. 16-19 of Ref. 1 can be written as

$$
\begin{array}{ll}
R_{1}=3 g_{2} / A, & R_{2}=3\left(S_{\nu}-g_{0}\right) / A \\
R_{3}=3\left(S_{\mu}-g_{0}\right) / A, & R_{4}=3 g_{1} / A \\
R_{5}=3\left(3 g_{0}+g_{2}\right) / A ; & A=1-S_{\mu}-S_{\nu}+g_{0}
\end{array}
$$

where the second rank order parameters for the absorption and emission transition moments $S_{\mu}$ and $S_{\nu}$ are defined by

$S_{v}=\left\langle P_{2}\right\rangle ; \quad S_{\nu}=\left\langle P_{2}\right\rangle P_{2}\left(\cos \beta_{v}\right)$

Further

$g_{k}=g(k k 00) P_{2}\left(\cos \beta_{\nu}\right), k=0,1,2$

where

$g(k k 00)=\frac{1}{\tau_{\mathrm{F}}} \int_{0}^{\infty} G(k k 00 t) \exp \left(-t / \tau_{\mathrm{F}}\right) \mathrm{d} t$

and the correlation functions $G(k k 00 t)$ are given by

$G(k k 00 t)=\left\langle D_{k 0}^{2}\left(\Omega_{0}\right) D_{k 0}^{2 *}\left(\Omega_{\imath}\right)\right\rangle$

Here $\tau_{\mathrm{F}}$ is the isotropic fluorescence decay constant $(\approx 10 \mathrm{~ns}$ for $\mathrm{DPH}) . D_{m . n}^{2}$ are Wigner rotation matrix elements [4] and $\Omega_{0} \equiv\left(\alpha_{0} \beta_{0} \gamma_{0}\right)$ denotes the set of three Euler angles that transform the laboratory frame into the molecular frame at time $t=0$; $\Omega$, is defined analogously for time $t . P_{L}$ is the Legendre polynomial of order $L$. The angled brackets denote an ensemble average over a macroscopic volume element. It is important to realize that the ensemble average entails an average over all the independent probe molecule populations in the sample.

We note further that, as a consequence of our assumption that every population can be described 
by the same dynamic behaviour, the information about the populations will be contained only in the orientational order parameters $\left\langle P_{2}\right\rangle$ and $\left\langle P_{4}\right\rangle$.

The order parameter $\left\langle P_{2}\right\rangle$ can be obtained directly from the experimental data, as can the angle $\beta_{v}$. However, $\left\langle P_{4}\right\rangle$ and the information about the molecular dynamics is contained in the correlation functions $G(k k 00 t)$. The functions can only be evaluated in terms of a model for the reorientational motion of the molecule. We shall here consider only the strong collision [1,5-7] and the rotational diffusion [5-10] models.

\section{(a)Strong collision model}

The molecules are here assumed to undergo random rotational jumps, with a residence time $\tau_{0}$ at any orientation [1,5-7]. The correlation functions $G(k k 00 t)$ can be shown to decay exponentially as

$$
\begin{aligned}
G(k k 00 t)= & G(k k 000) \exp \left(-t / \tau_{0}\right)+\left\langle P_{2}\right\rangle^{2} \\
& \times\left[1-\exp \left(-t / \tau_{0}\right)\right] \delta_{k 0}
\end{aligned}
$$

Substitution of Eqn. 7 into Eqn. 5 leads to

$g(k k 00)=w_{0} G(k k 000)+\delta_{k 0}\left\langle P_{2}\right\rangle^{2}\left(1-w_{0}\right)$

with

$w_{0}=\tau_{0} /\left(\tau_{\mathrm{F}}+\tau_{0}\right)$

The values of $G(k k 000)$ have been tabulated in Refs. 5-7.

Within this model the system parameters $\left\langle\mathrm{P}_{2}\right\rangle$, $\left\langle\mathrm{P}_{4}\right\rangle$ and $w_{0}$ can be derived analytically from $R_{1}, \ldots, R_{5}[2,3]$.

\section{(b) Rotational diffusion model}

The molecule is assumed to undergo stochastic rotational diffusion subject to an anisotropic orienting potential $U(\beta)$, where $\beta$ is the angle between the local director (the normal to the bilayer surface) and the long molecular axis. The equation for the orientational conditional probability is [5-8]

$$
\frac{\partial}{\partial t} P\left(\Omega_{0} \mid \Omega t\right)=D_{\perp} \Gamma_{\Omega} P\left(\Omega_{0} \mid \Omega t\right)
$$

where $D_{\perp}$ is the perpendicular component of the rotational diffusion tensor $\boldsymbol{D}$ which is assumed to have cylindrical symmetry about the molecular axis. The stochastic operator $\Gamma_{\Omega}$ is given by

$$
\begin{aligned}
\Gamma_{S 2} P\left(\Omega_{0} \mid \Omega t\right)= & \nabla_{\Omega 2}^{2} P\left(\Omega_{0} \mid \Omega t\right) \\
& +(\sin \beta)^{-1} \cdot \frac{\partial}{\partial \beta}\left[\sin \beta P\left(\Omega_{0} \mid \Omega t\right) \frac{\mathrm{d}}{\mathrm{d} \beta} U(\beta)\right]
\end{aligned}
$$

and $\nabla_{\Omega}^{2}$ is the Laplacian operator written in terms of the Euler angles describing the orientation of the molecule with respect to the laboratory frame. The initial boundary condition is $P\left(\Omega_{0} \mid \Omega 0\right)=\delta(\Omega$ $\left.-\Omega_{0}\right)$. We shall here assume that $U(\beta)$ is cylindrically symmetric and is given by

$U(\beta)=\left\{\lambda_{2} P_{2}(\cos \beta)+\lambda_{4} P_{4}(\cos \beta)\right\} k T$

Expansion of $P\left(\Omega_{0} \mid \Omega t\right)$ in a basis set of Wigner rotation matrices transforms the differential equation into a matrix equation which is solved numerically.

The correlation functions $G(k k 00 t)$ can now be shown [5-8] to be described as a sum of exponential decays:

$$
G(k k 00 t)=\sum_{M} b_{M}^{k} \exp \left(-D_{\perp} \alpha_{M}^{k} t\right)
$$

where the decay amplitudes $b_{M}^{k}$ and the decay rates $\alpha_{M}^{k}$ are complex functions of $\lambda_{2}$ and $\lambda_{4}$ in Eqn. 14 and are obtained from the numerical solutions of the equation of motion. Within this model we finally obtain

$$
g(k k 00)=\sum_{M} \frac{b_{M}^{k}}{\left(1+D_{\perp} \tau_{F} \alpha_{M}^{k}\right)}
$$

In order to apply this model a non-linear leastsquares procedure is used to fit the experimental parameters $R_{1}, \ldots, R_{5}$. Here $\lambda_{2}, \lambda_{4}, D_{\perp} \tau_{\mathrm{F}}$ and $P_{2}\left(\cos \beta_{v}\right)$ are used as free model parameters. The order parameters $\left\langle P_{2}\right\rangle$ and $\left\langle P_{4}\right\rangle$ are determined by the coefficients $\lambda_{2}$ and $\lambda_{4}$. We note here that our choice of the orienting potential, Eqn. 12 spans the entire $\left\langle P_{2}\right\rangle,\left\langle P_{4}\right\rangle$ plane, Fig. 2. This plane is defined by the inequalities [1-3]: 
$-1 / 2 \leqslant\left\langle P_{2}\right\rangle \leqslant 1$

$1 \geqslant\left\langle P_{4}\right\rangle \geqslant\left(35\left\langle P_{2}\right\rangle^{2}-10\left\langle P_{2}\right\rangle-7\right) / 18$

$-3 / 7 \leqslant\left\langle P_{4}\right\rangle \leqslant\left(5\left\langle P_{2}\right\rangle+7\right) / 12$

The 'Wobbling-in-cone' model developed by Kinosita et al. [9] and discussed by Lipari and Szabo [10] is a special case of the diffusion model. Here the orienting potential $U(\beta)$ is assumed to have the form

$U(\beta)=0 \quad 0 \leqslant \beta<\beta_{0}$

$U(\beta)=\infty \quad|\beta| \geqslant \beta_{0}$

With this form of the orienting potential the values of $\left\langle P_{2}\right\rangle$ and $\left\langle P_{4}\right\rangle$ are uniquely determined by the value of $\beta_{0}[10]$. This relation defines a line in the $\left\langle P_{2}\right\rangle,\left\langle P_{4}\right\rangle$ plane, Fig. 2. It can be seen that the model predicts negative values for $\left\langle P_{4}\right\rangle$ if $0 \leqslant\left\langle P_{2}\right\rangle \leqslant 0.55$. We shall show below that this behaviour is inconsistent with our experiments. The relation between $\left\langle P_{2}\right\rangle$ and $\left\langle P_{4}\right\rangle$ for the Gaussian model proposed by Kinosita et al. [11] is also shown in Fig. 2. This model is equivalent to the one described above but with $\lambda_{4}=0$ in Eqn. 12 .

\section{Materials}

Dioleoylphosphatidylcholine (DOPC) and egg phosphatidylcholine were obtained from Sigma

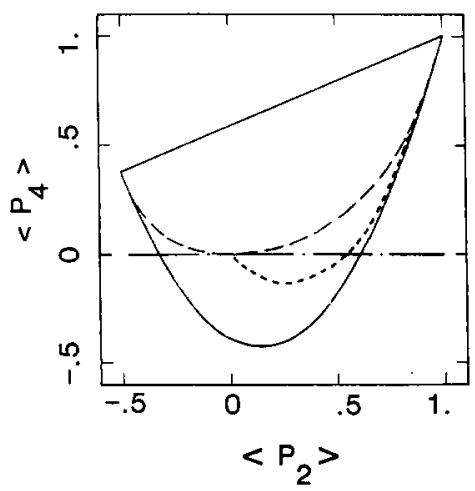

Fig. 2. The relation between $\left\langle P_{2}\right\rangle$ and $\left\langle P_{4}\right\rangle$. The continuous lines give the physical boundaries (see text). --- , the relation obtained from a gaussian orientational distribution function; - . - . -, the relation defined by the 'wobbling-in-cone' model; $-\cdot-$, is the $\left\langle P_{4}\right\rangle=0$ line. and used without further purification after chromatographical checks. Soybean phosphatidylcholine and digalactosyl diacylglycerol (DGDG) were prepared from soybeans [12] and spinach leaves [13], respectively, as described below. 1,6-Diphenyl-1,3,5-hexatriene (DPH) was purchased from Aldrich. Ethanol was obtained from Baker (AR). Doubly distilled water was used throughout.

All the preparative steps were carried out strictly under a nitrogen atmosphere. The lipids came into contact with air for a short time only during the alignment procedure, see below. The formation of peroxides was monitored following [20] by recording absorption spectra in the $200-300 \mathrm{~nm}$ region.

Purification of soybean lecithin. Crude soybean lecithin was bought from V.N.R. Reformproducten B.V., Ede, The Netherlands and prepurified by column chromatography on aluminium oxide 90 (Merck, Darmstadt, F.R.G.) using chloroform with increasing proportions of methanol as the eluent. The soybean lecithin fractions obtained were purified further by preparative high pressure liquid chromatography(HPLC) with the HPLC-equipment described in Ref. 12 or with home-built HPLC-equipment. As preparative chromatographic columns were used: (1) $500 \times 50 \mathrm{~mm}$ stainless steel (S.S.), packed with Polygosil 60-1525 Machery Nagel and Co, Düren, F.R.G.; (b) $250 \times$ $22.7 \mathrm{~mm}$ Lichrosorb Si-60-7 (S.S.) (Chrompack Nederland, Middelburg, The Netherlands). The columns were eluted with chloroform/methanol $(70: 30, \mathrm{v} / \mathrm{v})$. The fractions collected were analysed for purity on Silica gel 60 plates $(10 \times 10 \mathrm{~cm}$, for nano-TLC, art 5633, Merck, Darmstadt, F.R.G.). Chloroform/methanol/water $(65: 25: 4, v / v)$ was used as the eluting mixture. The fractions containing pure soybean phosphatidylcholine were dried and stored dry under $\mathrm{N}_{2}$ at $-20^{\circ} \mathrm{C}$.

Purification of DGDG from spinach. Total lipid extraction from spinach chloroplasts and subsequent fractionation in lipid classes was performed as described in Ref. 13. The acetone fraction obtained, containing mainly galactolipids, was purified further by using preparative HPLC as described above. A Lichrosorb Si-60-7 column (S.S., $250 \times 22.7 \mathrm{~mm}$ ) was used. The column was eluted with chloroform/methanol $(85: 15, \mathrm{v} / \mathrm{v})$. Analysis for purity was done as described above. We also 
used DGDG bought from Lipid Products (South Nutfield, U.K.). Analysis for purity was done as described. Only DGDG containing no contaminants by these criteria was used for our investigations.

Preparation of DPH-containing bilavers. $20 \mathrm{mg}$ lipid in ethanol solution were mixed with a $5 \cdot 10^{-4}$ $M$ solution of DPH in tetrahydrofuran. The final lipid to DPH molar ratio was $250: 1$. The samples were dried in the dark by flushing with nitrogen gas. Water was added to the dry material to form a $30 \%(w / w)$ mixture. The mixture was then allowed to equilibrate for $5 \mathrm{~h}$ at $35^{\circ} \mathrm{C}$ under a humidified nitrogen atmosphere.

Macroscopically oriented multibilayers were prepared by gently rubbing the hydrated lipid mixture between two microscope glass coverslips (thickness $175 \pm 5 \mu \mathrm{m}$ ). The alignment was carried out at room temperature $\left(25^{\circ} \mathrm{C}\right)$ and monitored by optical microscopy following Asher and Pershan [14]. On using this preparation method about $20 \%$ of the samples of DOPC and soybean phosphatidylcholine exhibited excellent alignment over areas larger than $1 \mathrm{~cm}^{2}$. These areas were selected for further experimental work. In marked contrast, well-aligned samples of DGDG and egg PC were produced consistently.

Well-aligned samples were sealed with a two component epoxy resin along their four rims in order to prevent dehydration. The contribution of scattered excitation or fluorescence light was minimized by completely blackening the samples except for a $5 \times 5 \mathrm{~mm}$ wide square area. The thicknesses of the samples, measured by dark field microscopy, were between 12 and $40 \mu \mathrm{m}$.

\section{Experimental}

Angle-resolved fluorescence depolarization (AFD) experiments were carried out at room temperature $\left(25^{\circ} \mathrm{C}\right)$ on a home-built fluorimeter. It was equipped with a water-cooled light-stabilized $1500 \mathrm{~W}$ Xe arc (Osram) and a Peltier cooled EMI 9816A or RCA 31034 PM tube, operating at approximately $-25^{\circ} \mathrm{C}$ and a cathode voltage of $-1200 \mathrm{~V}$. The angles $\theta$ and $\phi$ were set to within $1^{\circ}$ by two goniometers on which the sample holder and the PM tube assembly were independently mounted.
The necessary precautions to minimize systematic errors in the AFD experiments have been discussed in detail previously $[2,3]$.

DPH was excited at $365 \mathrm{~nm}$ with an FWHM (full width at half maximum) bandwidth of $8 \mathrm{~nm}$. The fluorescence was observed by means of interference filters, FWHM $12 \mathrm{~nm}$, at $432 \mathrm{~nm}$ for all lipids and additionally at 448 and $494 \mathrm{~nm}$ for egg phosphatidylcholine in order to check a possible dependence of the depolarization ratios on the emission wavelength of DPH. The intensity of the exciting light was kept as low as possible in order to avoid bleaching of the DPH molecules, yet at the same time maintaining a good signal-to-noise ratio. The intensity of the DPH fluorescence, measured at the same scattering geometry, remained constant during the experiment. Control experiments using well-aligned samples of pure lipids showed that the intrinsic fluorescence signals from the lipids and the coverslips amounted to less than $1 \%$ of the DPH fluorescence intensity.

The refractive indices of the membrane samples were determined to within $1 \%$ with a thermostated ABBE refractometer (Bleeker). These were required for applying optical corrections $[2,3]$.

\section{Results and Discussion}

\section{(i) Determination of $R_{1}-R_{5}$}

Angle-resolved fluorescence depolarization ratios were measured for DPH molecules embedded in bilayers of egg PC, soybean PC, DOPC and DGDG. The same values of the depolarization ratios, $R_{0}$ and $R_{\mathrm{e}}$, within the experimental error of $2 \%$ were obtained on rotating the samples by an arbitrary angle about the normal to their surfaces. This indicates that the macroscopic distribution of DPH molecules is uniaxially symmetric. Furthermore, different samples of the same lipid system yielded reproducible results within experimental error.

The results obtained from egg PC bilayers were similar to those found earlier in our laboratory for bilayers of saturated phosphatidylcholines above the phase transition of the chains [2,3]. However, in marked contrast, a quite different angular dependence of $R_{0}$ and $R_{\mathrm{e}}$ were observed in the other bilayer systems which contained predominantly unsaturated lipid molecules. 
The experimental results, corrected for refraction and transmission losses at the air/glass and glass/ sample interfaces [1-3], were analysed following the procedure described by us previously $[2,3]$. This can be summarised as follows:

(1) $R_{1}$ and $R_{2}$ are determined from the linear dependence of $R_{0}$ on $\sin ^{2} \theta$, Eqn. 1a.

(2) $R_{1}$ and $R_{2}$ are also determined from the 'extraordinary' experiment with $\theta=0$. Eqn. $1 \mathrm{~b}$ then yields

$1 / R_{\mathrm{e}}=\left(1+R_{1}\right) /\left(1-R_{1}\right)+\left[\left(R_{2}-R_{1}\right) /\left(1-R_{1}\right)\right] \sin ^{2} \phi$

$1 / R_{\mathrm{e}}$ is now linear in $\sin ^{2} \phi$.

(3) $R_{1}$ and $R_{3}$ are determined from the "extraordinary' experiment on setting $\phi=0$. Eqn. 1b can now be written as

$\frac{1}{\left(1 / R_{\mathrm{e}}\right)-1}=\frac{R_{3}+1}{2 R_{1}} \tan ^{2} \theta+\frac{1-R_{1}}{2 R_{1}}$

This experiment is particularly useful if the angle between the absorption and emission moments within the probe molecule is not known: the difference $R_{2}-R_{3}$ is a measure of this angle. Only if $R_{2}=R_{3}$ are the absorption and emission moments parallel.

(4) $R_{4}$ and $R_{5}$ are obtained from the "extraordinary' experiment on varying both $\theta$ and $\phi$. In the general case Eqn. $1 \mathrm{~b}$ can be cast as

$$
\begin{aligned}
Y(\theta, \phi) & \equiv a_{1}(\theta) / R_{\mathrm{e}}-\mathrm{a}_{2}(\theta) \\
& =A(\theta) \sin ^{2} \phi+B(\theta) \sin 2 \phi
\end{aligned}
$$

with

$a_{1}(\theta)=1-R_{1}+\left(R_{3}+R_{1}\right) \sin ^{2} \theta$

$a_{2}(\theta)=1+R_{1}+\left(R_{3}-R_{1}\right) \sin ^{2} \theta$

and

$A(\theta)=R_{2}-R_{1}+R_{5} \sin ^{2} \theta$

$B(\theta)=R_{4} \sin 2 \theta$

As $R_{1}, R_{2}, R_{3}$ are known from the previous analysis, the coefficients $a_{1}$ and $a_{2}$ can be evaluated at any angle $\theta_{0}$. The coefficients $A\left(\theta_{0}\right)$ and $B\left(\theta_{0}\right)$ are then obtained from a least-squares fit to Eqn. $18 \mathrm{c}$. By repeating this procedure for all the values of $\theta_{i}$, a set of numbers $A\left(\theta_{i}\right)$ and $B\left(\theta_{i}\right)$ is obtained. Finally, values of $R_{4}$ and $R_{5}$ are extracted from the linear dependence of $A\left(\theta_{i}\right)$ and $B\left(\theta_{i}\right)$ on $\sin ^{2} \theta_{i}$ and $\sin 2 \theta_{i}$, respectively (Eqns. $18 \mathrm{~d}$ ).

This procedure suffers from the drawback that the accuracy of the values of $R_{4}$ and $R_{5}$ is largely determined by errors in $R_{1}, R_{2}$ and $R_{3}$. In order to avoid this problem we have also used a non-linear optimalization procedure for the determination of $R_{1}, \ldots, R_{5}$ from Eqn. $1 \mathrm{~b}$.

It must be emphasized that the angles $\theta$ and $\phi$ appearing in Eqns. 1 and 18 are both angles measured within the sample.

The experimental results for soybean PC and egg $P C$ are shown in Figs. 3 and 4 and in Figs. 5 and 6 , respectively. The values of $R_{1}, \ldots, R_{5}$ for all the systems studied are summarized in Table $I$ under A. Figs. 3b, c, d and $5 \mathrm{~b}, \mathrm{c}, \mathrm{d}$ show plots of $Y(\theta, \phi)$, Eqn. $18 \mathrm{c}$, as a function of $\phi$ for a number of values of $\theta$. It is important to note that the $Y(\theta, \phi)$ axes in the figures are scaled differently for the various values of $\theta$, as given in the legends. Consequently, the experimental errors (2\%) shown, appear larger for the lower values of $\theta$. Figs. 4 and 6 show that the derived values of $A(\theta)$ and $B(\theta)$, Eqn. $18 \mathrm{~d}$ closely follow the predicted angular de-
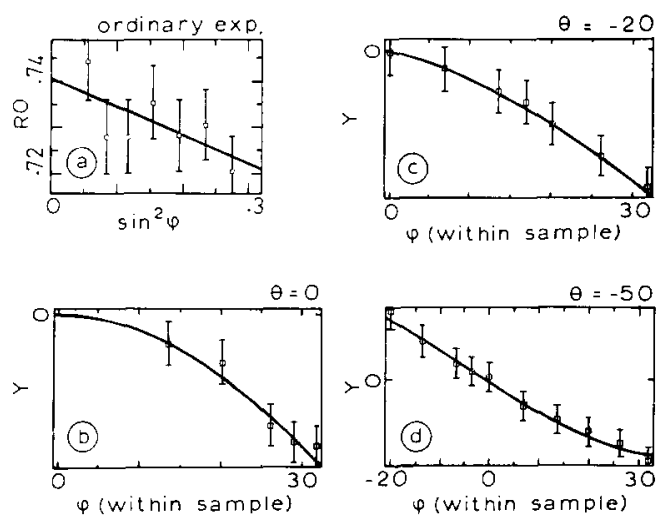

Fig. 3. Theoretical fits of AFD results for a soybean PC/water $70: 30(\mathrm{w} / \mathrm{w})$ bilayer system with $4 \cdot 10^{-3} \mathrm{M} \mathrm{DPH}$ at $25^{\circ} \mathrm{C}$. $\Phi:$ experimental values, relative accuracy $2 \%$. Solid lines indicate least-squares fits according to Eqns. 1a and 18. (a) Ordinary experiment; (b), (c), (d) extraordinary experiment. Vertical scales $\theta=0^{\circ}: 0.10 ; \theta=-20^{\circ}: 0.14$ and $\theta=-50^{\circ}: 0.30$. 

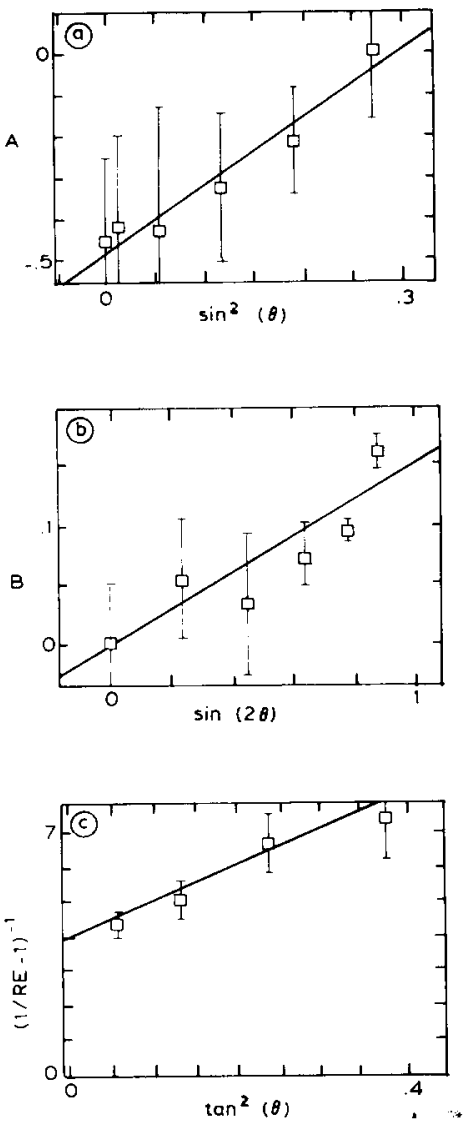

Fig. 4. AFD results for soybean PC. (a) $A\left(\theta_{i}\right)$ obtained from extraordinary experiments, as a function of $\sin ^{2} \theta_{i}$, Eqn. $18 \mathrm{~d}$. (b) $B\left(\theta_{i}\right)$ as a function of $\sin 2 \theta_{i}$, Eqn. $18 \mathrm{~d}$. (c) The determination of $R_{3}$ from Eqn. $18 \mathrm{~b}$. Note that if $R_{3}=R_{2}$ the slope would be three times smaller than that obtained.

pendence. Furthermore, the results, Figs. 4 and 6 , show unequivocally that $R_{2} \neq R_{3}$, thus indicating that the emission moment of DPH is tilted relative to the molecular symmetry axis. A similar behaviour had been observed by us in systems of saturated lipids, with or without cholesterol $[2,3]$.

The values of $R_{1}$ and $R_{2}$ obtained from the 'ordinary' and 'extraordinary' experiments were found to be in good agreement within experimental error.

(ii) $\left\langle P_{2}\right\rangle$ and $\beta_{\nu}$

The order parameters $S_{\mu}$ and $S_{\nu}$ of the absorption and emission moments, respectively, can be obtained directly from $R_{1}, \ldots, R_{5}$, as can the values of $g_{k}, k=0,1,2[1-3]$
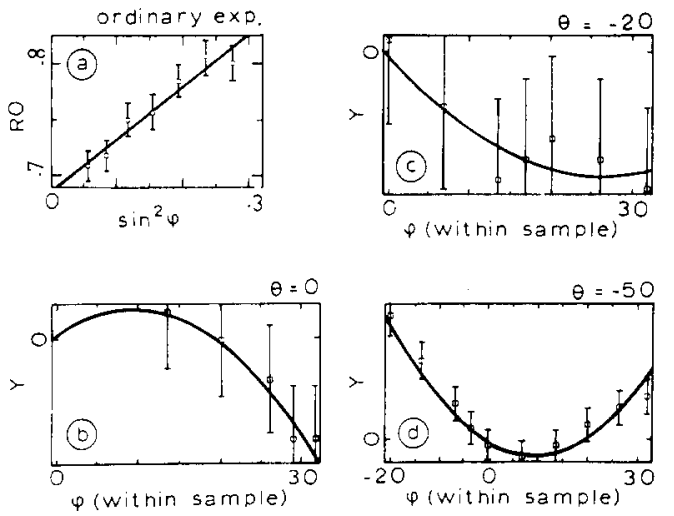

Fig. 5. Theoretical fits of $\mathrm{AFD}$ results for an egg $\mathrm{PC} /$ water $70: 30(\mathrm{w} / \mathrm{w})$ bilayer system with $4 \cdot 10^{-3} \mathrm{M} \mathrm{DPH}$ at $25^{\circ} \mathrm{C}$. $\Phi$ : experimental values, relative accuracy $2 \%$. Solid lines indicate least-squares fits according to Eqns. 1a and 18. (a) Ordinary experiment, (b), (c), (d) extraordinary experiments. Vertical scales $\theta=0^{\circ}: 0.054 ; \theta=-20^{\circ}: 0.045$ and $\theta=-50^{\circ}: 0.27$.
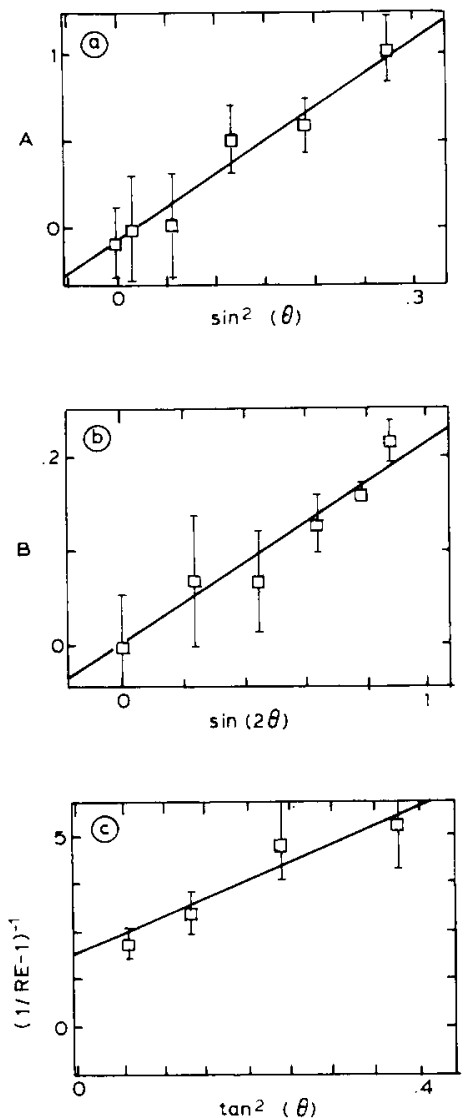

Fig. 6. AFD results for egg PC. (a) $A\left(\theta_{i}\right)$ obtained from extraordinary experiments, as a function of $\sin ^{2} \theta_{i}$, Eqn. $18 \mathrm{~d}$. (b) $B\left(\theta_{i}\right)$ as a function of $\sin 2 \theta_{i}$, Eqn. $18 \mathrm{~d}$. (c) The determination of $R_{3}$ from Eqn. $18 \mathrm{~b}$. Note that if $R_{3}=R_{2}$, the slope of the line would be half that obtained. 
TABLE I

EVALUATION OF $R_{1}-R_{5}$ FOR ORIENTED LIPID MEMBRANE SYSTEMS

(A) Values obtained from a non-linear least-squares analysis of the experimental data. (B) Best fit values obtained from the rotational diffusion model. (C) Best fit values obtained from the 'wobbling-in-cone' model.

\begin{tabular}{|c|c|c|c|c|c|c|}
\hline \multicolumn{2}{|l|}{$\begin{array}{l}70: 30(w / w) \\
\text { lipid/water } \\
\text { mixture with } \\
4 \cdot 10^{-3} \mathrm{M} \mathrm{DPH} \mathrm{in}\end{array}$} & $R_{1}$ & $R_{2}$ & $R_{3}$ & $R_{4}$ & $R_{5}$ \\
\hline \multirow[t]{3}{*}{ Soybean PC } & A & 0.111 & -0.333 & 0.920 & 0.143 & 1.17 \\
\hline & B & 0.12 & -0.13 & 0.88 & 0.11 & 1.2 \\
\hline & $\mathrm{C}$ & 0.12 & 0.04 & 0.77 & 0.26 & 0.68 \\
\hline \multirow[t]{3}{*}{ DGDG } & A & 0.230 & -0.339 & 0.451 & 0.306 & 1.81 \\
\hline & B & 0.21 & -0.19 & 0.43 & 0.23 & 1.9 \\
\hline & C & 0.25 & -0.03 & 0.28 & 0.47 & 1.3 \\
\hline \multirow[t]{3}{*}{ DOPC } & A & 0.181 & -0.441 & 0.382 & 0.154 & 1.72 \\
\hline & B & 0.19 & -0.25 & 0.36 & 0.12 & 1.8 \\
\hline & $\mathrm{C}$ & 0.20 & -0.07 & 0.24 & 0.33 & 0.94 \\
\hline \multicolumn{7}{|l|}{ Egg PC } \\
\hline \multirow[t]{3}{*}{$\lambda_{\mathrm{em}}=432 \mathrm{~nm}$} & A & 0.165 & 0.138 & 1.43 & 0.202 & 3.46 \\
\hline & B & 0.147 & 0.138 & 1.42 & 0.209 & 3.47 \\
\hline & C & 0.141 & 0.221 & 1.52 & 0.502 & 1.41 \\
\hline \multirow[t]{3}{*}{$\lambda_{\mathrm{em}}=448 \mathrm{~nm}$} & A & 0.178 & -0.0014 & 1.39 & 0.181 & 3.47 \\
\hline & B & 0.161 & -0.0014 & 1.38 & 0.186 & 3.47 \\
\hline & C & 0.203 & 0.0016 & 0.44 & 0.408 & 1.10 \\
\hline \multirow[t]{3}{*}{$\lambda_{\mathrm{em}}=494 \mathrm{~nm}$} & A & 0.168 & 0.071 & 1.04 & 0.196 & 3.28 \\
\hline & B & 0.164 & 0.071 & 1.04 & 0.197 & 3.28 \\
\hline & $\mathrm{C}$ & 0.170 & 0.126 & 0.953 & 0.465 & 1.22 \\
\hline
\end{tabular}

In all the systems studied here $S_{\mu}>S_{\nu}$, so that $\beta_{v}>0^{\circ}$ (see Eqn. 3), and thus the absorption and emission moments are not mutually parallel. This is in agreement with earlier results $[2,3]$. The values of $\left\langle P_{2}\right\rangle=S_{\mu}$ are given in Table II.

Values for $\beta_{v}$ were obtained from $S_{v}$ and $\left\langle P_{2}\right\rangle$, Eqn. 3. The angle of $\approx 36^{\circ}$ calculated for DPH in egg PC is comparable to those found earlier $[2,3]$ for DPH in bilayers of saturated lipids. In contrast, the low values of $S_{\nu}$ obtained for the other bilayer systems yield $\beta_{v} \approx 50^{\circ}$. Such an angle, however, would yield much lower values for the $g_{k}$ 's, Eqn. 4, than are in fact observed. The calculations presented below indicate that an angle of $\approx 40^{\circ}$ is necessary to describe the experimental results. This inconsistency could well arise if the assumption of effective molecular cylindrical sym-
TABLE II

THE SECOND AND FOURTH RANK ORIENTATIONAL ORDER PARAMETERS $\left\langle P_{2}\right\rangle$ AND $\left\langle P_{4}\right\rangle, w_{0}$ AND THE ROTATIONAL CORRELATION TIME $\tau_{0}$ AS OBTAINED FROM THE STRONG COLLISION MODEL

\begin{tabular}{lcccc}
\hline $70: 30(\mathrm{w} / \mathrm{w})$ & $\left\langle P_{2}\right\rangle$ & $\left\langle P_{4}\right\rangle$ & $w_{0}$ & $\tau_{0}{ }^{a}(\mathrm{~ns})$ \\
lipid $/$ water & & & & \\
mixture with & & & & \\
$4 \cdot 10^{-3} \mathrm{M}$ DPH in & & & & \\
\hline Soybean PC & 0.32 & 0.24 & $>0.95$ & $\gg 10$ \\
DGDG & 0.23 & 0.12 & $>0.95$ & $\gg 10$ \\
DOPC & 0.26 & 0.31 & $>0.95$ & $\gg 10$ \\
Egg PC & & & & \\
$\lambda_{\text {em }}=432 \mathrm{~nm}$ & 0.45 & 0.39 & 0.56 & 13 \\
$\lambda_{\mathrm{em}}=448 \mathrm{~nm}$ & 0.45 & 0.45 & 0.68 & 21 \\
$\lambda_{\mathrm{em}}=494 \mathrm{~nm}$ & 0.40 & 0.36 & 0.53 & 11 \\
\hline
\end{tabular}

${ }^{a}$ Here we have assumed $\tau_{\mathrm{F}}=10 \mathrm{~ns}$. 
metry is no longer valid as a consequence of a lower rate of reorientation about the long axis of the molecule. A description of the experiment in terms of non-cylindrically symmetric molecules may thus be needed. This requires the introduction of extra order parameters and correlation times into the calculations $[1,15]$, so that more than five model parameters must now be derived from the values of $R_{1}, \ldots, R_{5}$. In view of this we have not attempted such an analysis of the experimental data.

\section{(iii) $\left\langle P_{4}\right\rangle$ and reorientational dynamics}

The information about $\left\langle\mathrm{P}_{4}\right\rangle$ and the reorientational motion of the molecules is contained in the three functions $g_{0}, g_{1}$ and $g_{2}$. As discussed above these have been interpreted on the basis of three models for the reorientational motion of the molecules.

The results obtained from the strong collision model, using Eqns. 2-9 are given in Table II. We note here again that in the theoretical derivation of Eqns. $1-5, \beta_{\nu}$ was taken to be a molecular property independent of the orientational dynamics of the probes. It can be seen that the order parameters $\left\langle P_{2}\right\rangle$ and $\left\langle P_{4}\right\rangle$ are of the same magnitude for all the bilayer systems. However, significant differences are found in the values of $w_{0}$. This parameter must lie in the range $0 \leqslant w_{0} \leqslant 1$. The values of $w_{0}$ for egg PC bilayers lie within this range and on taking $\tau_{\mathrm{F}}=10 \mathrm{~ns}$ [16], yield rotational correlation times of 10-20 ns for the DPH molecules. These values, again, are similar to those obtained for
DPH in bilayers of saturated lipids [2,3].

On the other hand, the results for soybean PC, DGDG and DOPC show that $w_{0}>0.95$ and thus that $\tau_{0} \gg \tau_{\mathrm{F}}$. Consequently these systems are effectively frozen on the fluorescence time scale and the values of the correlation functions at $t=0$. $G(k k 000)$, determine the order parameters $\left\langle P_{2}\right\rangle$ and $\left\langle P_{4}\right\rangle$. Furthermore, we find that the experimental values of the functions $g_{0}, g_{1}$ and $g_{2}$ are only consistent with $\beta_{v} \approx 35-45^{\circ}$, in contrast with the values of $\beta_{v}$ obtained from $S_{v}$. These values of $\beta_{v}$ are comparable with those of $[2,3]$ and with that of $\approx 36^{\circ}$ obtained for egg phosphatidylcholine. The results again suggest that the assumption of effective cylindrical symmetry for the DPH molecules breaks down in the unsaturated systems.

We have also analysed the experimental results in terms of the rotational diffusion model with $D_{\perp} \tau_{\mathrm{F}}, \lambda_{2}, \lambda_{4}$ and $P_{2}\left(\cos \beta_{v}\right)$ as free model parameters (see above). Eqns. 4 and 14 show that in this case products of the form

$P_{2}\left(\cos \beta_{v}\right) \sum_{M} b_{M}^{k} /\left(1+D_{\perp} \tau_{\mathrm{F}} \alpha_{M}^{k}\right)$

appear in the expressions for the functions $g_{k}$. The best-fit values for $R_{1}, \ldots, R_{5}$ are given in Table $\mathrm{I}$ under $\mathrm{B}$ and the values of the corresponding model parameters $\lambda_{2}, \lambda_{4}, D_{\perp} \tau_{\mathrm{F}}$ and $P_{2}\left(\cos \beta_{\nu}\right)$ are shown in Table III. It can be seen from Table I that the fits for egg PC bilayers are in excellent agreement with the experimental values. The agreement for the other systems is less satisfactory and in partic-

TABLE III

EVALUATION OF THE PARAMETERS, $\lambda_{2}, \lambda_{4}, D_{\perp} \tau_{F}$ AND $P_{2}\left(\cos \beta_{v}\right)$ OBTAINED FOR THE ROTATIONAL DIFFUSION MODEL

\begin{tabular}{|c|c|c|c|c|c|c|}
\hline $\begin{array}{l}70: 30(\mathrm{w} / \mathrm{w}) \\
\text { lipid/water } \\
\text { mixture with } \\
4 \cdot 10^{-3} \mathrm{M} \mathrm{DPH} \mathrm{in}\end{array}$ & $\lambda_{2}$ & $\lambda_{4}$ & $\left\langle P_{2}\right\rangle$ & $\left\langle P_{4}\right\rangle$ & $D_{\perp} \tau_{\mathrm{F}}$ & $P_{2}\left(\cos \beta_{\nu}\right)$ \\
\hline Soybean PC & -0.57 & -2.1 & 0.30 & 0.33 & $\lll 1$ & 0.20 \\
\hline DGDG & -0.70 & -1.4 & 0.26 & 0.23 & $\lll 1$ & 0.39 \\
\hline DOPC & -0.20 & -2.7 & 0.24 & 0.37 & $\ll 1$ & 0.32 \\
\hline \multicolumn{7}{|l|}{ EGG PC } \\
\hline$\lambda_{\mathrm{em}}=432 \mathrm{~nm}$ & -1.4 & -1.2 & 0.45 & 0.28 & 0.12 & 0.49 \\
\hline$\lambda_{\mathrm{em}}=448 \mathrm{~nm}$ & -1.2 & -1.7 & 0.45 & 0.34 & 0.08 & 0.44 \\
\hline$\lambda_{\mathrm{em}}=494 \mathrm{~nm}$ & -1.2 & -1.3 & 0.40 & 0.28 & 0.12 & 0.54 \\
\hline
\end{tabular}


ular significantly different values are obtained for $R_{2}$. This is again a consequence of the low values of $S_{\nu}$ in these systems.

We have been unable to obtain satisfactory fits for any of the bilayer systems by setting $\lambda_{4}=0$ in the model calculations. The introduction of an extra term $P_{6}(\cos \beta)$ into the orienting potential, Eqn. 14, produced no significant improvement in the fits and the low values of $\left|\lambda_{6}\right| \leqslant 0.05$ indicated that this term is not in fact important. The discrepancies between the experimental and best fit values for $R_{1}, \ldots, R_{5}$ for bilayers of soybean $\mathrm{PC}$, DOPC and DGDG may well be alleviated by the introduction of non-cylindrically symmetric terms into the orienting potential. As a check of the model calculations we note that the values of $\left\langle P_{2}\right\rangle$ are in excellent agreement with those of $S_{\mu}$ for all the systems as are the values of the product $\left\langle P_{2}\right\rangle P_{2}\left(\cos \beta_{v}\right)$ and $S_{v}$ for egg PC, Eqn. 3.

The calculations indicate further that the experimental results for the other bilayer systems can be well described if $\beta_{\nu}$ lies in the range $35^{\circ}-45^{\circ}$. This is in good agreement with the values obtained from the strong collision model.

The order parameters $\left\langle P_{2}\right\rangle$ and $\left\langle P_{4}\right\rangle$ obtained from the rotational diffusion model indicate that significant though qualitative differences exist in the orientational statistics of the DPH molecules in the systems studied here. The same conclusion is also reached on the basis of the strong collision model. A comparison of Tables II and III shows further that similar values of $\left\langle P_{4}\right\rangle$ are obtained from both models.

Although the DPH molecules appear to have similar static orientational properties in all the bilayer systems studied here, they exhibit marked differences in their reorientational dynamics. The calculations indicate that in bilayers of soybean PC, DOPC and DGDG the DPH molecules are effectively stationary on the time scale of the fluorescence decay as $D_{\perp} \tau_{\mathrm{F}} \ll 1$. On the other hand, the DPH molecules undergo a fairly rapid reorientational diffusion with $D_{\perp} \sim(1-2) \cdot 10^{7} \mathrm{~s}^{-1}$ in egg $\mathrm{PC}$ bilayers. This result is in agreement with the conclusion drawn from the strong collision model.

The agreement in the results obtained from the Strong Collision and Rotational Diffusion models is gratifying and the reason can be found in the time dependence of the correlation functions
$G(k k 00 t)$. Inspection of Eqns. 7 and 13 shows that since $w_{0}>0.95$ and $D_{\perp} \tau_{\mathrm{F}} \ll 1$, for bilayers of soybean PC, DGDG and DOPC, the correlation functions do not appreciably decay on the time scale defined by the fluorescence life time of DPH. Thus, the values of the correlation functions at time $t=0$ effectively determine the order parameters $\left\langle P_{2}\right\rangle$ and $\left\langle P_{4}\right\rangle$. As the correlation functions at $t=0$ are strictly model independent [1-3,5-7]. both models should then yield the same order parameters. The observed differences between the results obtained with the two models simply reflect the difference in the numerical procedures used to evaluate the model parameters. The calculations for egg PC bilayers using the Rotational Diffusion model show that the decay of each correlation function $G(k k 00 t), k=0,1,2$, is dominated by a single exponential component. This is in agreement with the theoretical considerations of Zannoni [5-7]. Thus, in this case, the differences between our two models are to be found only in the values of the correlation times assigned to the correlation functions. For the strong collision model we find $\tau_{0} \approx 13 \mathrm{~ns}$, while the three correlation times yielded by the Rotational Diffusion model, Eqn. 13, are $1 /\left(D_{\perp} \alpha^{\circ}\right) \approx 28 \mathrm{~ns}, 1 /\left(D_{\perp} \alpha^{1}\right)$ $\approx 9 \mathrm{~ns}$ and $1 /\left(D_{\perp} \alpha^{2}\right) \approx 13$ ns. Here we have taken $\tau_{\mathrm{F}} \approx 10 \mathrm{~ns}$. Thus, since both models yield correlation times of the same order of magnitude, they also yield similar values for the order parameters $\left\langle P_{2}\right\rangle$ and $\left\langle P_{4}\right\rangle$.

We have also analysed our experiment with the 'wobbling-in-cone' model using the analytical expressions obtained by Lipari and Szabo [10]. Here we have taken $\cos \beta_{0}, D_{\mathrm{w}} \tau_{\mathrm{F}}$ and $P_{2}\left(\cos \beta_{\nu}\right)$ as free model parameters. The best-fit values for $R_{1}, \ldots, R_{5}$ are given in Table $I$ under $C$ and the values of the corresponding parameters are shown in Table IV. It can be seen from Table I that the fits are much less satisfactory than those obtained with the Rotational Diffusion model. In particular, no satisfactory fits could be obtained for egg PC bilayers with the 'wobbling-in-cone' model, in contrast to the other two models used here. We have also attempted to fit the experimental data directly with this model. However, the values for the model parameters obtained were the same as those from the fitting of the $R$ 's. Table IV shows further that the values of $\left\langle P_{2}\right\rangle,\left\langle P_{4}\right\rangle$ and $D_{\mathrm{w}} \tau_{\mathrm{F}}$ yielded by this 


\section{TABLE IV}

EVALUATION OF THE PARAMETERS $\left\langle P_{2}\right\rangle,\left\langle P_{4}\right\rangle, D_{\mathrm{w}} \tau_{\mathrm{F}}$ AND $P_{2}\left(\cos \beta_{\nu}\right)$ OBTAINED FOR THE 'WOBBLING-INCONE' MODEL

\begin{tabular}{|c|c|c|c|c|c|}
\hline $\begin{array}{l}70: 30(w / w) \\
\text { lipid/water } \\
\text { mixture with } \\
4 \cdot 10^{-3} \mathrm{M} \mathrm{DPH} \mathrm{in}\end{array}$ & $\beta_{0}$ & $\left\langle P_{2}\right\rangle$ & $\left\langle P_{4}\right\rangle$ & $D_{\mathrm{w}} \tau_{\mathrm{F}}$ & $P_{2}\left(\cos \beta_{v}\right)$ \\
\hline Soybean PC & $69.2^{\circ}$ & 0.24 & -0.13 & $\ll 1$ & 0.23 \\
\hline DGDG & $73.7^{\circ}$ & 0.18 & -0.11 & $\ll 1$ & 0.49 \\
\hline DOPC & $76.8^{\circ}$ & 0.14 & -0.09 & $\ll 1$ & 0.38 \\
\hline \multicolumn{6}{|l|}{ Egg PC } \\
\hline$\lambda_{\mathrm{cm}}=432 \mathrm{~nm}$ & $59.7^{\circ}$ & 0.38 & -0.12 & $\ll 1$ & 0.33 \\
\hline$\lambda_{\mathrm{em}}^{\mathrm{em}}=448 \mathrm{~nm}$ & $72.1^{\circ}$ & 0.20 & -0.12 & $\ll 1$ & 0.41 \\
\hline$\lambda_{\mathrm{em}}=494 \mathrm{~nm}$ & $65.7^{\circ}$ & 0.29 & -0.13 & $\ll 1$ & 0.37 \\
\hline
\end{tabular}

model are inconsistent with the results obtained from the other models discussed above. The reason for the poor fits can be traced back to an unsatisfactory feature of the 'wobbling-in-cone' model. As can be seen from Fig. 2, the model constrains $\left\langle P_{4}\right\rangle$ to take negative values in the range of $\left\langle P_{2}\right\rangle$ involved here. Note that the value of $\left\langle P_{2}\right\rangle$ is strictly model independent, Eqns. 3-5. In the lipid systems studied by us thus far $\left\langle P_{4}\right\rangle$ has always been found to take on positive values. It is interesting that this model yields satisfactory fits in systems with a high degree of orientational order $[2,3]$ where it predicts positive values for $\left\langle P_{4}\right\rangle$. We are therefore, forced to conclude that the "wobbling-in-cone' model is unsuitable for the analysis of the reorientational dynamics of DPH molecules in our bilayer systems.

\section{(iv) Interpretation of the order parameters}

The orientational ordering of the DPH molecules in lipid bilayers can be fully described in terms of an orientational distribution function $f(\beta)$ in which $\beta$ is the angle between the bilayer and the molecular symmetry axes $[1-3,5-7,15]$. In its turn $f(\beta)$ is characterized by an infinite set of order parameters $\left\langle P_{2}\right\rangle,\left\langle P_{4}\right\rangle, \ldots,\left\langle P_{L}\right\rangle, L$ even, which are the averages of Legendre polynomials of order $L$ over the distribution function. Consequently, the interpretation of the order parameters will depend on the assumed functional form of $f(\beta)$. We have previously argued [1-3] that a useful estimate of $f(\beta)$ can be obtained if both $\left\langle P_{2}\right\rangle$ and $\left\langle P_{4}\right\rangle$ are
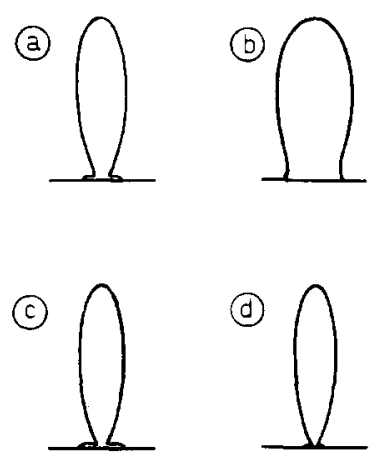

Fig. 7. Orientational distribution functions $f(\beta)$ for $\mathrm{DPH}$ molecules in (a) soybean PC, (b) DGDG, (c) DOPC and (d) egg PC. The lipid to water ratio was $70: 30(w / w)$ for all samples.

known and if $f(\beta)$ is reconstructed on the basis of an information-theoretic approach. The essential point here is that given $\left\langle P_{2}\right\rangle$ and $\left\langle P_{4}\right\rangle$ the most probable values of $\left\langle P_{L}\right\rangle$ 's, $L \geqslant 6$, are calculated under the constraint that the informational entropy of $f(\beta)$ is a maximum. The resulting distribution function has the form [18]

$f(\beta)=A \exp \left\{\lambda_{2} P_{2}(\cos \beta)+\lambda_{4} P_{4}(\cos \beta)\right\}$

where $A$ is a normalization constant and $\lambda_{2}$ and $\lambda_{4}$ are determined from the known values of $\left\langle P_{2}\right\rangle$ and $\left\langle P_{4}\right\rangle$. This distribution function corresponds to a Boltzmann distribution with the angle-dependent orienting potential of Eqn. 12.

Fig. 7 shows the distribution functions for soybean PC, DGDG, DOPC and egg PC reconstructed using Eqn. 19 with the values of $\left\langle P_{2}\right\rangle$ and $\left\langle P_{4}\right\rangle$ given in Table II. It can be seen that most of the DPH molecules are preferentially aligned perpendicular to the bilayer surface. Nevertheless, in soybean PC, DGDG and DOPC bilayers a small, but significant, fraction of the molecules lie parallel to the plane of the bilayers. Similar distribution functions for DPH have been obtained by us in systems of saturated lipids above the phase transition $[2,3]$.

\section{(v) Conclusions}

The results presented above show that although the DPH molecules appear to have similar static orientational properties in all the bilayer systems studied here, they exhibit marked differences in 
their reorientational dynamics. The introduction of unsaturation into the lipid chains seems to cause a slowing down in the dynamics of the DPH molecules. This conclusion is based on an analysis of the experimental data in which the DPH molecules are assumed to possess an effective cylindrical symmetry.

We have encountered internal inconsistencies in analysing the results for soybean PC, DOPC and DGDG bilayers on taking the DPH molecules to be cylindrically symmetric. On the other hand, this model can be successfully used in analyzing angle-resolved experiments on DPH embedded in bilayers of egg PC and saturated phosphatidylcholines with or without cholesterol [2,3]. Our experiments strongly suggest that DPH molecules in bilayers of unsaturated lipids lose their effective cylindrical symmetry as a result of a lower rate of reorientation around their long axes. The fluorescence depolarization experiment senses this mode of motion as the absorption and emission transition moments are not mutually parallel. This conclusion is based on the observation that $R_{2} \neq$ $R_{3}$, so that $S_{\mu}>S_{v}$ as discussed above. Unfortunately, a description of the data in terms of non-cylindrically symmetric molecules requires more model parameters than the five quantities yielded by our steady-state experiments.

Corroborative evidence for our conclusion can, however, be found in an earlier study of DPH molecules in vesicles of unsaturated lecithins using time-resolved techniques [17] This study revealed that the time-dependent fluorescence anisotropy, $r(t)$, decays appreciably on the time scale of the fluorescence life time $\tau_{\mathrm{F}}$. Yet the analysis of our experimental data in terms of cylindrically symmetric molecules leads to the opposite conclusion. The discrepancy can be resolved simply if the DPH molecules no longer possess and effective cylindrical symmetry. Furthermore the experimental results obtained from vesicle systems seem to be poorly described by a model based on the assumption of cylindrically symmetric DPH molecules. An examination of Fig. 1 of Ref. 17 reveals large and systematic discrepancies between the experimental and theoretical curves for $r(t)$; the differences are masked to some extent by the use of a logarithmic scale. Nevertheless we note that the parameters derived from our experiments re- produce the values for the steady-state anisotropy $r^{s}$, Eqn. 5 of Ref. 17 and the cone angles quoted for the same systems.

Our suggestion that DPH molecules in unsaturated lipid bilayer systems cannot be treated as having effective cylindrical symmetry is contrary to the assumptions made in Ref. 17. We emphasize, however, that our angle-resolved experiments on macroscopically oriented samples are more discriminating than experiments on vesicles as they afford the direct determination of the time-averaged correlation functions $g_{0}, g_{1}$ and $g_{2}$ as well as the order parameters of the absorption and emission dipole moments. Such details cannot be obtained from experiments on macroscopically isotropic vesicle systems.

The results presented above are at odds with the generally accepted picture that unsaturation in the lipid chains causes the rates of reorientational motion to increase. However, studies in our laboratory of the motion of cholestane spin-label molecules incorporated into DGDG bilayers [19] are in agreement with the fluorescence depolarization experiments.

One conclusion to be drawn from our work is simply that DPH molecules are not suitable probes for monitoring bilayers of unsaturated lipids.

\section{References}

1 Van der Meer, B.W., Kooyman, R.P.H. and Levine, Y.K. (1982) Chem. Phys. 66, 39-50

2 Kooyman, R.P.H., Vos, M. and Levine, Y.K. (1983) Chem. Phys. 81, 461-472

3 Vos, M., Kooyman, R.P.H. and Levine, Y.K (1983) Biochem. Biophys. Res. Commun. 116, 462-468

4 Rose, M.E. (1957) Elementary theory of angular momentum, J. Wiley, New York

5 Zannoni, C. (1979) Mol. Phys. 38, 1813-1827

6 Zannoni, C. (1981) Mol. Phys. 42, 1303-1320

7 Zannoni, C., Arcioni, A. and Cavatorta, P. (1983) Chem. Phys. Lipids 32, 179-250

8 Nordio, P.L., Rigatti, G. and Segre, U. (1973) Mol. Phys. $25,129-136$

9 Kinosita, K., Kawato, S. and Ikegami, A. (1977) Biophys. J. 20, 289-305

10 Lipari, G. and Szabo, A. (1981) J. Chem. Phys. 75, 2971-2976

11 Kinosita, K., Ikegami, A. and Kawato, S. (1982) Biophys. J. 37, 461-464

12 Geurts van Kessel, W.S.M., Tieman, M. and Demel, R.A. (1981) Lipids 16, 58-63 
13 Fork, D.C., Van Ginkel, G. and Harvey, G. (1981) Plant Cell Physiol. 22, 1035-1042

14 Asher, S.A. and Pershan, P.S. (1979) Biophys. J. 16, 393-421

15 Zannoni, C. (1979) Ch. 3 in The molecular physics of liquid crystals (Luckhurst, G.R. and Gray, G.W., eds.), Ch. 3, pp. 51-83, Academic Press, New York

16 Chen, L.A., Dale, R.E., Rogh, S. and Brand, L. (1977) J. Biol. Chem. 252, 2163-2169
17 Stubbs, C.D., Kouyama, T., Kinosita, K., Jr. and Ikegami. A. (1981) Biochemistry 20,4257-4262

18 Levine, R.D. and Tribus, M. (eds.) (1979) The maximum entropy formalism, M.I.T. Press, Cambridge

19 Koole, P. (1984) Ph.D. Thesis, University of Utrecht

20 Klein, R.A. (1970) Biochim. Biophys. Acta 210, 486-489 